\title{
Young Children's Comprehension of Modal Expressions
}

\author{
James P. Byrnes \\ Michelle A. Duff
}

University of Michigan

\begin{abstract}
The present study assessed children's knowledge of how speakers use modal auxiliaries such as might and have to to indicate their degree of belief in the truth of their statements (epistemic modality), and to socially regulate behavior (deontic modality). We also considered whether modals comprise a semantic system that is progressively attained in a way similar to other semantic systems. Children aged 3 to 5 years were given two tasks: one that assessed comprehension of modal expressions for the epistemic domain (here, the spatial location of objects), and one that assessed comprehension of such expressions for the deontic domain (i.e., permission for the restrictions on behavior). Results showed that even 3year-olds comprehend partial aspects of meaning of epistemic expressions considered individually. Development occurred primarily between the ages of 3 and 4 in the ability to make judgments of the relative strength and synonymy of pairs of modal expressions. These results, which show a progressive attainment of the meaning of modals, indicate metacognitive and social cognition ability not usually attributed to preschoolers.
\end{abstract}

A modal expression is a statement that contains a modal auxiliary such as must, may, or could that modifies or qualifies the propositional content of this statement (Lyons, 1977). For example, in the expression "It may be Tuesday," the modal may qualifies the proposition It is Tuesday. More specifically, modals are used by speakers to convey their degree of belief in the truth of the propositional content of their utterance. A speaker who says "It has to be Tuesday" expresses a strong belief in the truth of this expression, whereas the use of may as in "It may be Tuesday" signals less subjective certainty about the truth of the expression to the listener. Modals, then, are selected by speakers in the context of considering the state of their knowledge. Such a consideration, however, requires the ability to self-reflect. Therefore, systematic modal use in spontaneous

The authors wish to express sincere gratitude to Drs. Jean Berko Gleason, Brian MacWhinney, and Catherine Snow for making additional data available to us. We also thank Jodi DeSantis for help in running subjects.

Correspondence and requests for reprints should be sent to James P. Bymes, Institute for Child Study, Department of Human Development, University of Maryland, College Park, MD 20742. 
discourse, or, equivalently, knowledge of how modals are used, can be very informative about an individual's ability to reflect upon his or her own knowledge. It is in this way that knowledge of modals can provide an important index of self-reflective or metacognitive ability.

Additionally, modals also serve an important function in the social regulation of behavior. They are used within statements uttered by persons of authority to either place restrictions on or give permission for behavior (Lyons, 1977). For example, a parent might say "You have to sit down" to place restrictions on a child's behavior. In this way, comprehension of modals plays an important role in early social cognition. In the present study, we assessed preschoolers' knowledge of how speakers use modals to convey the strength of their belief and to regulate behavior.

The modal auxiliary system is complex and consists of multiple components that children eventually come to master. Before describing age trends in the acquisition of modals, we first describe four key aspects of this system that children need to come to know. With such a theoretical framework in place, it becomes possible to address hypotheses regarding the order of acquisition of the four aspects.

Similar to word classes such as antonyms (e.g., big/small) and temporal adverbs (e.g., always/never), modals appear to form a semantic system (cf. Clark, 1972; Kuczaj, 1975). By semantic system it is meant that terms in a word class are linked within a network by virtue of sharing common meaning components. For example, all temporal adverbs have in common a meaning component regarding frequency of occurrence. Modals, on the other hand, all convey something about the necessity or possibility of something being the case (PierautLeBonniec, 1980; von Wright, 1951). In particular, to believe in the necessity of something is to believe that it certainly must be true. Thus, being certain about a statement is equivalent to a belief in its necessity. In English, certainty or necessity is conveyed by must and have to (e.g., "It has to be Tuesday"). Uncertainty, on the other hand, relates more closely to possibility than to necessity. In particular, if two or more possibilities exist and a speaker does not know which in fact is true, then the speaker adopts an attitude of uncertainty. In English, speakers convey their uncertainty about multiple possibilities by using may, might, or could. For example, a speaker who is unsure what day of the week it is would say "It might be Tuesday." Additionally, necessity relates to possibility in that knowledge of only one possibility yields a sense of certainty or necessity, whereas knowledge of more than one possibility yields a sense of uncertainty (Byrnes \& Overton, 1986). Thus, the conceptual basis for comprehending modals consists primarily of knowledge of possibilities. When children become capable of conceiving of possibilities and can reflect on how many of these possibilities could be true, they can convey this knowledge using modals.

In addition to shared meaning components, each of the antonym, temporal 
adverb, and modal semantic systems can also be characterized by an internal arrangement of opposites. Thus, we have big versus small, always versus never, and must versus must not as examples for each system, respectively. The modal system differs from the antonym and temporal adverb systems by the fact that single terms often exist for opposites in the latter two systems whereas typically no such single terms exist for the modal system. Thus, we have small instead of not big in the antonym system but must not for must in the modal system. Regardless, it is evident that negation plays a fundamental role in all such systems in terms of the construction of opposites. Thus, the cognitive basis for comprehending modals also entails knowledge of how negation participates in the construction of opposites. 1

The modal and temporal adverb systems are similar along a third dimension that may contribute to developmental trends. In these systems, degrees of opposition exist. More specifically, the terms can be arranged in an ordinal fashion with opposites found at points that are symmetrical with respect to a midpoint. For example, temporal adverbs can be arranged in an ascending order of frequency: never, rarely, sometimes, usually, and always. With sometimes as the midpoint, we have always and never as symmetrical opposites and rarely and usually as symmetrical opposites. This arrangement reflects a continuum of frequency.

An analogous arrangement exists for modal terms. Modality can be thought of as a continuum of beliefs or attitudes arranged in the following ascending ordinal fashion, with example terms for each attitude in parentheses: strong disbelief (can't), disbelief (might not), indeterminacy (I don't know), belief (might), strong belief (have to). Similar to temporal adverbs, a midpoint and symmetrical opposites exist. With I don't know as the midpoint, we have have to and can't as symmetrical opposites and might and might not as symmetrical opposites. For example, "It can't be Tuesday" is a symmetrical opposite for "It has to be Tuesday," as is "It might not be Tuesday" for "It might be Tuesday." An appropriate expression for the midpoint would be "I don't know whether it's Tuesday or not." This continuum of attitudes expresses relative degrees of conviction towards an expression. Thus, a speaker who says "It has to be Tuesday" expresses a stronger degree of conviction in knowing what day it is than a speaker who says "It might be Tuesday." Both, however, express a belief in it being Tuesday. Conversely, a speaker who says "It can't be Tuesday" expresses a stronger degree of conviction in his or her knowledge than a speaker who says "It might not be Tuesday." However, this time they both express a belief in it not being Tuesday. Finally, a speaker who has no idea what day it is

1 We should note that the role of negation here is a novel theoretical assumption we make. The relation between negation, opposites, and modals has not been demonstrated to be a unitary process. In the absence of theoretical counterproposals or falsifying empirical data, we retain this assumption given that it is hard to imagine one creating multiple possibilities from some proposition $p$ without negation; that is, possibility arises out of knowing that either $p$ or not-p could be true. 
should use none of the four possible terms if none of the days is judged to be more likely. Rather, a more appropriate response would be "I don't know." Besides knowledge of possibilities and the role of negation, then, the cognitive basis for comprehending modals also consists of refining attitudes towards possibilities into a continuum of personal conviction. Thus, children need to know that has to is stronger than might in the belief direction and can't is stronger than might not in the disbelief direction.

A final aspect of the modal system children need to master concerns the fact that adult speakers in most languages use the same modal terms or affixes to express two kinds of modality: epistemic and deontic (Lyons, 1977; Shepard, 1982). Epistemic modality is concerned with matters of knowledge or belief about certain states of affairs (Lyons, 1977, p. 703). For example, modalized expressions regarding object properties and categories fall in the domain of epistemic modality, as do those concerning spatial, temporal, or logical relations. Thus, "It must be a dog" (categorical relation), "It has to be under the cup" (spatial relation), and "He may be the first one" (temporal relation) are example expressions for epistemic modality. Epistemic modality concerns those concepts typically studied by cognitive developmentalists. As a speech act, an expression of epistemic modality functions as what Searle (1983) labels "representative": that is, an attempt to inform the listener of the speaker's beliefs about the way things are.

Deontic modality, on the other hand, is concerned with the necessity or possibility of acts performed by morally responsible or socially regulated agents (Lyons, 1977, p. 823). Statements of deontic modality concern the regulation of acts of the listener in terms of either giving permission for or placing restrictions on behavior. For example. "You have to eat your dinner" and "You can play now" express deontic modality. In order for a statement of deontic modality to be appropriately uttered as a speech act, however, the speaker must be in a power relation to the listener; that is, the speaker must have the authority to give permission or prohibit actions and must be seen in this way by the listener. These expressions function as what Searle (1983) labels "directives"; that is, attempts by the speaker to get the listener to do something.

In sum, there are four aspects of the modal system that may contribute to developmental patterns of acquisition. First, since modals appear to cohere into a semantic system, their acquisition pattern may be similar to that found for other semantic systems. It is often the case for semantic systems that children initially learn only partial aspects of meaning for individual terms and later acquire meaning components that appropriately differentiate the terms (cf. Clark, 1972; Kuczaj, 1975). Second, acquisition of the modal system requires comprehension of how negation participates in the construction of opposites. It may be that initially only some of the opposite relations are established and the rest are established progressively. Third, the opposite relations need to be arranged into a continuum of degrees of belief. It may be that a global comprehension of how 
negation creates opposites precedes the construction of a continuum of symmetrical opposites. This trend is likely, given that both the antonym and temporal adverb systems follow this acquisition pattern. Finally, children need to extend the modal semantic system to both the epistemic and deontic domains in order to use modals in the way adult speakers do. Linguists suggest that since deontic modality concerning the regulations of acts is more primitive and salient to young children, it is probably learned earlier than epistemic modality (Lyons, 1977; Shepard, 1982). The historical development of modals in several languages appears to support this claim.

Given this description of the modal system, we can now consider developmental research on the topic. Unfortunately, there exist few studies that address children's comprehension of modals specifically in terms of these four aspects. In studies of children's spontaneous productions, for example, the incidence of modals was not a primary concern. These studies can only inform us as to when modals first appear and which ones appear first. These studies found that can and could are usually the earliest modals to appear in children's natural discourse and are first produced when children are between the ages of 2 and 3 years (Fletcher, 1985; Kuczaj \& Maratsos, 1983; Shepard, 1982). Shepard (1982) examined protocols of a single child and reported that modals are used deontically before they are used epistemically.

A recent experimental study specifically considered the issues of degree of conviction and extension to epistemic and deontic domains. Hirst \& Weil (1982) assessed the ability of 3-to 6-year-olds to make strength comparisons among is, may, should, and must sentences. For the epistemic domain, children were asked to decide between two sentences constructed with these terms to help them find a concealed object. For example, children heard one puppet say "The peanut must be under the cup" and another say "The peanut should be under the box." For each sentence pair, the peanut could be found in the location indicated by the sentence containing the stronger of the two modals. The results showed that it was not until children were in the age range of $4 ; 0-4 ; 6$ that an above-chance number were able to make a distinction between is and the three modals, and not until the age range of 5;6-6;0 that children could discriminate among the three modals in terms of strength.

For the deontic domain, children were required to make distinctions among must, may, and should, but when considering sentences that directed a doll to go to one of two small-scale rooms. After hearing a sentence pair such as "You must go to the green room" and "You may go to the red room," children had to indicate where the doll would go by pointing. The results again showed that it was not until children were in the age range of $5 ; 6-6 ; 0$ that a significant number could make the appropriate strength distinctions among the modals.

In sum, whereas spontaneous production studies seem to suggest a much earlier onset, the experimental findings suggest that significant aspects of the system are not understood until age 5 or 6 . However, over $50 \%$ of 3 - and 4-year- 
olds have been found to demonstrate significant insight into the formally similar temporal adverb system (Kuczaj, 1975). Additionally, Hirst \& Weil's (1982) findings run contrary to those of Shatz, Wellman, and Silber (1983) regarding children's production of mental verbs such as know and think. These terms, like modals, indicate children's assessment of their own or another's knowledge. Using rigorous coding procedures, these authors found that $43 \%$ of children in the age range of $3: 7-3 ; 11$ appropriately use mental verbs to refer to mental states.

The primary purpose of the present study was twofold. The first was to clarify the age trends regarding when children appear to comprehend significant aspects of modals. The second was to explicitly consider the hypothesis that children first acquire partial aspects of the meanings of modal terms and later acquire a more complete understanding. None of the studies described above can be used to address this issue. We modified Hirst \& Weil's (1982) procedure to accomplish these aims.

The first modification concerned Hirst \& Weil's choice of modal terms. While it is possible that children at times use must, may, and should in natural discourse, the published data as well as our own corpus collected for another study (Byrnes \& Duff, 1988) suggest that preschoolers are unlikely to use these more formal terms. In our own data, for example, children were more likely to use can $(56 \%)$, have to $(23 \%)$, and could $(9 \%)$. No instances of either must or may were found. We found a similar pattern when we analyzed a sample of utterances from Gleason, Perlmann, and Greif's (1984) study made available. to us through the Child Language Data Exchange System (see MacWhinney \& Snow, 1985). In Gleason et al. (1984), preschoolers conversed with their parents over dinner. An examination of the productions made by 10 of their children aged 3;0-5;7 revealed that $93 \%$ of modals were can, has to, could, and might. Moreover, $95 \%$ of the modals that parents expressed to children were these informal terms. Thus, perhaps Hirst \& Weil underestimate children's ability due to the use of terms that are less familiar to preschoolers.

A second modification concerned the issue of partial meaning. We included single-sentence trials in which children did not have to contrast degrees of strength but had to simply use modals to guide their search. Both have to and might share a meaning component in terms of belief in what is stated, but differ in degree of strength. Similarly, both can't and might not share a meaning component in terms of disbelief in what is stated, but differ in degree of strength. Performance on single-sentence trials can be used to see if terms that share meaning components guide a child's search in similar ways. Performance on these trials can be compared to that on trials that contrast degree of belief to see if the partial, shared meaning of these terms is acquired prior to the full meaning.

A third modification considered the issue of synonymy. Because negation figures prominently in the modal system, it is possible to derive synonymous expressions for a given belief essentially through a double-negation procedure. 
However, the method only works when there are only two possibilities. Consider a case in which a penny is hidden under one of either a blue or a red cup. One creates synonymy by first pairing a modal expression and its symmetrical opposite (e.g. . "It has to be under the red cup" and "It can't be under the red cup"). Then one replaces the location of the penny in one of the pair with the opposite location, effectively negating it internally (e.g., "It can't be under the blue cup"). At best, however, these expressions are only referentially synonymous. We were nevertheless interested in children's comprehension of the referential synonymy of these expressions because mental processing of these pairs can be thought to place demands on the cognitive system of opposites. Flavell (1985) notes how young children's concepts are often "fragile" to the extent that skills can only be exposed when task demands are minimized. We employed synonymous pairs to assess the internal integrity or "fragility" of children's modal system.

Finally, we were interested in how performance compared across the epistemic and deontic domains. We modified Hirst and Weil's procedure to consider the issue of whether children have an easier time comprehending modal terms in one domain over the other.

\section{METHOD}

\section{Subjects}

Fifty-four preschoolers participated. There were 20 three-year-olds (mean $=3$ years, 8 months; range $2 ; 10-3 ; 11$ ), 15 four-year-olds (mean $=4$ years, 8 months; range $4 ; 0-4 ; 11$ ), and 19 five-year-olds (mean $=5$ years, 7 months; range $5 ; 1-6 ; 3)$. Approximately half of the sample was male and half was female. The children were recruited from a middle-class preschool of a large midwestern university. In order to be included in the study, children had to pass a pretest for colors. Only one child was dropped as a result of not knowing the four colors employed.

\section{Design}

All children were given the epistemic task and deontic task in a random order. It was usually necessary to conduct the testing over two to three sessions due to the youngest children's limited attention spans.

\section{Tasks and Procedure}

Epistemic Task. This task consisted of three white poster boards with pairs of plastic drinking cups tumed upside down at fixed locations. A penny was placed under one of the two cups at each location. The three boards corresponded to the three kinds of items: single-sentence, strength contrast, and synonymous items. 
For single-sentence items, red and blue cup-pairs were positioned in a leftright zig-zag pattern. The colors and positions of the pennies were randomly alternated to counteract position or color biases in our youngest subjects. A lamb puppet was used to "whisper a hint " into the child's ear that would indicate the placement of the penny. The experimenter placed the puppet close to the child's ear, but spoke normally in order to "whisper." Children were told that the puppet would help them find the pennies. To increase motivation, children were told that they would be given a sticker if they found the penny frequently. Prior to beginning the trials, children were given a two-trial pretest to ensure they would use the puppet's statements to guide their search. Here the puppet said, "It's under the blue cup" on the first trial and "It's under the red cup" in the second. Children were to indicate the location of the penny either by pointing or saying the color. All children easily passed both trials of the pretest.

There were 16 single-sentence trials consisting of four instances of each of four sentences:

(a) It has to be under the $X$ (red/blue) cup.

(b) It can't be under the $X$ (red/blue) cup.

(c) It might be under the $X$ (red/blue) cup.

(d) It might not be under the $X$ (red/blue) cup.

Thus, there was one item expressing necessity (i.e., has to), one expressing impossibility (i.e., can't), and two expressing possibility (i.e., might, might not). Of note is the fact that the informal might was used instead of the more formal may to parallel using have to and can't instead of must. Additionally, two items contained negation and two items did not. To be scored as correct, a child had to recognize that has to and might indicate that the penny was under the cup named (e.g., blue for "It has to be under the blue cup") whereas can' $t$ and might not indicate the penny was under the cup not named (e.g., blue for "It can't be under the red cup'). Whereas it is logically possible that the penny can be found under the cup not named for has to and might and the cup named for can't and might not, the "correct" response corresponded to the pragmatic implications of the utterance. According to Conversational Postulates (Grice, 1975), a speaker should not try to lead the listener astray. Therefore, a listener should assume that the speaker has some idea where the penny is when he or she uses any of the four modal terms. A speaker who has no idea of where the penny could be should use "I don't know" in order to honestly convey lack of knowledge. Additionally, subjects were told that the puppets knew where the penny was. Thus, children received a score of $0-4$ for comprehending the pragmatic implications of each kind of item. For each test trial, the experimenter would point to the next pair of cups with "OK, let's try these (point) two cups" and would then say the next test sentence.

For strength contrast items, two lamb puppets were employed to say pairs of sentences. The puppets looked similar, except that one was gray and the other 
was white. For the second board, four red and blue cup-pairs were placed in the comers. A "road" was drawn down the center of the board and four "driveways" were drawn from the road to each of the cup-pairs. The children were told that a penny was hidden under one of the cups and that the way to find it was to listen to both of the puppets. Analogous to a task used by Scholnick \& Wing (1988), they were told that they were going to walk down the roads with the puppets and "visit the houses." As a pretest, both puppets said the same sentence in sequence for two trials. For example, on the first pretest trial, after one puppet said "It's under the blue cup" into one ear, the other said this same sentence into other ear. For the second pretest trial, both said "It's under the red cup" in sequence. Stickers were again used for incentive; all children passed the pretest.

The strength contrast test trials consisted of a total of eight sentence pairs. There were four trials each of:

(a) It has to be under the $X$ (e.g., red) cup

vs.

It might be under the non-X (e.g., blue) cup.

(b) It can't be under the $X$ (e.g., red) cup

vs.

It might not be under the non-X (e.g., blue) cup.

These pairs contrast degrees of conviction on the part of the speaker. In the has to vs. might pair, the child must choose between absolute conviction towards the necessity of $p$ (i.e., has to) and relative conviction towards the possibility of not$p$ (i.e., might). Thus, the child was required to opt for the stronger of the two. In the can't vs. might not pair, the child must choose between the impossibility of $p$ (i.e., can't) and the relative unlikelihood of not-p (i.e., might not). For example, for the pair "It can't be under the red cup" and "It might not be under the blue cup," children should look under the blue cup. These latter pairs require children to choose to follow the more strongly negative of the two sentences, whereas the other pairs require children to follow the more strongly positive of the two sentences. Children could receive a score of $0-4$ for each of the two kinds of strength contrast pairs.

The third kind of epistemic task items were synonymous items. The materials and procedure for these sentence pairs were identical to that for strength contrast pairs except that green and yellow cups were used. Additionally, the same four sentence frames used in the strength contrast items were regrouped to form two kinds of synonymous pairs:

(a) It has to be under the X (e.g., green) cup $=$ It can't be under the not-X (e.g., yellow) cup

(b) It might be under the $X$ (e.g., green) cup = It might not be under the non-X (e.g., yellow) cup 
To gain points for these pairs, children had to see that each sentence in a given pair indicated the same location of the penny. They could receive a score of $0-4$ for each of the two kinds of synonymous pairs.

Deontic Task. Four stories were used to determine children's comprehension of modal terms in the deontic domain. In each story, the main character (a child) expressed a desire to perform an act and the character's parents used a modal expression that either permitted or prohibited this act. The stories are presented in Table 1.

Six or seven line drawings were constructed to illustrate each story. Before starting a given story, the experimenter would array the pictures upside down in a row. As she told the story, she would progressively turn over the card that illustrated that particular part of the story. To assess children's comprehension of deontic modal expressions, the experimenter would ask the child to predict the character's actions immediately after those parts of the story in which a parent stated a modal expression. For example, in the "Johnny" story shown in Table 1, the experimenter would reach the line where the mother said, "You have to stay in" and would then ask the subject to predict the character's action with "What do you think Johnny did?" Up to that point, the first three pictures had been turned over and the child had to determine what was shown on the picture corresponding to the character's actions after hearing the modal expression. After the child made a prediction, the experimenter would resume the story with "Let's see what happens in the rest of the story." When she reached that part of the story in which the father stated a modal expression, the experimenter would again ask the subject to predict the child's actions. The order of stories was randomized.

Comprehension of deontic modal expressions requires knowledge of the social relations involved in acts of giving permission or restricting behavior; that is, what "being allowed to" or "not being allowed to" means. To make the contexts maximally familiar to children, we selected four highly desirable activities for young children and had the character's parents give permission or place restrictions. We also had children consider one parent's expression at a time. Having them consider both sentences makes the task one of deciding who wielded more power rather than one of comparing the relative strength of the expressions themselves. Hirst and Weil's (1982) deontic task, in contrast, consisted of a non-desirable activity (i.e., going to a room) requested in immediate sequence by power figures who are less salient to young children than parents (i.e., teachers). Whereas it would be useful to assess children's differential responses to speakers of various levels of power, we were not concerned with this factor in the present study.

Correct predictions served as indices of comprehension of modal expressions. As Lyons (1977, p. 824) notes, deontic modality always refers to future acts; that is, conformity or nonconformity with a deontic expression always tasks place in 


\section{Coincide}

Johnny (strong $=$ strong)

One day Johnny wanted to go outside and play. He 1

looked outside and saw all the children playing. 2

He said to his mom, "I want to go outside." 3

His mom said, "You have to stay in." *

Johnny was very sad.

The next day he said to his dad, "Dad, I want to go outside."

His dad said, "You can't go out."* 5

Johnny said. "O.K., I"ll stay in." 6

Mary (weak $=$ weak)

One day Mary went to a baseball game. She wanted to 1

$\begin{array}{ll}\text { watch the game but she was too small. } & 2\end{array}$

She said, "Mom, I want to stand up so I can see." 3

Her mom said, "You can stand up." *

Mary stood up for a while.

Then a little later she said, "Dad, I want to stand so I can see."

Her dad said, "You don't have to sit down." *

Mary liked watching the game.

\section{Conflict}

Josh (strong vs. weak)

One day Josh was playing outside. He was having a lot of fun. He wanted to stay out for a while but it was dinner time.

His mom said, "You have to come in."* 2

Josh came in.

The next day, Josh was playing again. He saw his dad come out to call him.

Josh said, "Dad, I want to stay and play."

His dad said, "You can stay out." *

Josh was so happy.

Sarah (weak vs. strong)

One night, Sarah wanted to watch TV shows. She wanted 1

to stay up like her older brother. 2

She said, "Mom, I want to stay up and watch TV." 3

Her mom said, "'You can stay up." *

She was so happy.

A little later her dad came in the room. Sarah said, "I 5

want to stay up and watch TV."

Her dad said, "You have to go to bed." 6

Sarah went to bed.

Note: * means a prediction was requested. 
the future. Thus, predictions of future acts serve as appropriate indices of comprehension. To gain points for each prediction, children had to correctly reconcile the character's desire with the parent's statement. When the parent restricted an activity, the correct response was the character's compliance. When the parent gave permission, the correct response was the character's compliance. When the parent gave permission, the correct response concerned the character's engaging in the desired activity (see Table 1). The character's desires were explicitly stated in the story and illustrated with pictures.

As shown in Table 1 , there were two kinds of stories as determined by whether the parent's statements coincided or conflicted with each other. The expressions that coincided were approximately synonymous by virtue of the addition of negation and substitutions of can for have to forms. They were analogous, though not identical, to the synonymous items for the epistemic task. In the present task, one sentence was not said immediately after the other as in the epistemic task. In the "Johnny" story, both expressions are equally strong: One expressed the necessity of an action and the other expressed the impossibility of its negation. However, they were not perfectly synonymous because one imposed a restriction not in place and the other released a restriction that was apparently already in place. In the "Mary" story, both expressions were equally non-binding: One expressed the possibility of an action and the other expressed the non-necessity of its negation. Children could receive a score of $0-4$ for the two coincide stories.

For the sentences in the two conflict stories, children should recognize that different predictions should be made due to differences in both content and degree of conviction. In the "Josh" story, one expresses the necessity of an action and the other expresses the possibility of its negation. In the "Sarah" story, one expresses the possibility of an action and the other expresses the necessity of its negation. The sentence pairs in these stories were analogous, though not identical, to the strength contrast pairs in the epistemic task. Children could receive a score of $0-4$ for the two conflict stories.

\section{RESULTS}

The results are presented by task. All significant main effects and interactions are interpreted using Newman-Keuls post-hoc tests.

\section{Epistemic Task}

We performed four analyses to assess developmental differences in comprehension of epistemic modal expressions: one for single-sentence items, one for strength contrast pairs, one for synonymous pairs, and one considering strength contrast and synonymous pairs together.

For the single-sentence items, a 3 (age: $3,4,5$ ) $\times 4$ (item: can't, has to, might, might not) ANOVA with repeated measures on the second factor was 
computed. Contrary to Hirst and Weil's (1982) finding for sentence pairs, this analysis revealed no main effects for either age, $F(2,51)=2.43$, n.s., or item, $F$ $(6,153)=0.82$. The age $\times$ item interaction was also not significant, $F(6,153)=$ 0.51 . Here, children at every age performed quite well ( $M^{\prime}$ 's $=3$-year-olds, $15.20 ; 4$-year-olds, $14.68 ; 5$-year-olds, 15.68 ) on every modal expression ( $M$ 's $=$ can't, 3.85; has to, 3.85; might, 3.80; might not, 3.71). Of note is the fact that the presence of negation in can't and might not did not hinder performance. Children knew, then, that "It can't be under the red cup" and "It might not be under the red cup" both indicate that the penny was under the blue cup. Thus, it can be at least said that children's similar responses for has to and might suggest they consider these terms to share a meaning component (both refer to the cup named), and similar responses for can't and might not suggest they consider these terms to share a meaning component (both refer to the cup not named).

A second analysis considered whether children could make more fine distinctions regarding relative strength. Recall that on strength contrast pairs, children heard two sentences and had to recognize that has to expresses stronger belief than might, and that can't expresses stronger disbelief than might not. Good performance on strength contrast pairs would suggest that these terms have been placed along a continuum; that is, a refined semantic system appears to be evident. A 3 (age: $3,4,5$ ) $\times 2$ (item: has to vs. might, can't vs. might not) ANOVA with repeated measures was computed for strength contrast pairs. This analysis revealed significant main effects of age, $F(2,51)=17.98, p<.001$, and item type, $F(1.51)=7.28, p<.01$. The age $\times$ item type interaction was not significant. For the main effect of age, the 5-year-olds $(M=7.05)$ and 4-yearolds $(M=6.27$ ) both performed significantly better than the 3-year-olds ( $M=$ 4.35). The older two groups didn't differ. Thus, it would appear that a continuum within the modal system initially emerges between the ages of 3 and 4 . In support of this claim, the percentages of children who were above chance on the strength contrast pairs ( 7 out of $8, p=.03$ ) were $10 \%, 47 \%$, and $84 \%$ for the $3-$, 4-, and 5-year-olds, respectively. Such percentages are higher than those found by Hirst and Weil (1982) using must, may, and should.

For the main effect of item, children performed significantly better on the has 10 vs. might contrast ( $M=3.13$ ) than on the can't vs. might not contrast $(M=$ 2.70). Thus, children had an easier time judging relative strength on the belief side of the midpoint of the continuum than on the disbelief side. That is, subjects found it easier to comprehend strength contrasts when the speaker seemed to know where the penny was likely to be than when the speaker seemed to know where the penny was unlikely to be.

A third analysis considered children's ability to judge the referential synonymy of sentence pairs. Recall that synonymy was achieved by pairing symmetrical opposites (e.g. . has to and can't) and negating the location of the penny in one of these sentences. These items required mental computation with negation and were employed to test the internal stability of the modal semantic 
system. A 3 (age: $3,4,5) \times 2$ (item: has to be $X=$ can't be not- $X$, might be $X=$ might not be not-X) ANOVA with repeated measures was computed for the synonymous pairs. This analysis revealed significant main effects of age, $F$ $(2.51)=5.15, p<.01$, and item, $F(1,51)=4.25, p<.05$. The age $\times$ item interaction was not significant. For the main effect of age, the 5-year-olds $(M=$ 7.84) performed significantly better than either the 4-year-olds $(M=6.87)$ or 3 year-olds $(M=6.45)$. The younger two groups didn't differ. Thus, it would appear that the 5-year-olds were better able to handle the cognitive demands of the synonymous pairs. However, children at all ages performed quite well. In fact, $60 \%, 67 \%$, and $100 \%$ of the 3-, 4-, and 5-year-olds, respectively, were above chance ( 7 out of 8 pairs, $p=.03$ ) on the synonymous pairs.

For the main effect of item, children performed significantly better on the has to be $X=$ can't be not- $X$ synonymous pair $(M=3.67)$ than on the might be $X=$ might not be not-X synonymous pair $(M=3.39)$. These findings would imply that children had an easier time computing synonymy with the two extreme terms of the continuum that were symmetrical opposites. The symmetrical opposites might and might not fall between the midpoint and extremes of the continuum.

A fourth analysis considered whether the above findings were simply due to an increased facility with negation with age. Here, the two strength contrast pair items and the two synonymous pair items were all considered in the same analysis: A 3 (age ) $\times 4$ (item: has to vs. can't, can't vs. might not, has to be $X$ $=$ can't be not- $X$, might be $X=$ might not be not-X) ANOVA with repeated measures. This analysis revealed main effects of age and item, as well as an age $x$ item interaction. The main effect of age revealed a monotonic increase with age. The age $\times$ item interaction resulted from there being no age differences for the synonymous has to $=$ can't $^{\prime}$ pair, but differing age patterns for the other three items.

For the main effect of item, performance was best on the synonymous has to $=$ can't pair $(M=3.66)$. The means for this item differed significantly from all other means. Performance was next best on the synonymous might $=$ might not pair $(M=3.39)$ and the strength contrast pair has to vs. might $(M=3.13)$, which didn't differ. Performance was worst on the strength contrast pair, can't vs. might not $(M=2.70)$.

If this item effect was simply due to facility with negation, the order should have been: (a) has to vs. might (no negatives) easiest; (b) might $=$ might not and has to $=$ can't (one negative) in the middle; and (c) might not vs. can't (two negatives) hardest. However, this order was not obtained. Additionally, there were no differences for the single-sentence items regardless of negation. Rather, the difficulty lies in terms of the kind of comparison made - that is, strength versus synonymy - and on whether strength of belief or strength of disbelief was being considered.

In sum, the findings for the single-sentence trials and the strength contrast trials taken together largely mirror those for antonyms and temporal adverb 
systems. In particular, children appear to acquire partial aspects of meaning first, and later refine the meaning of these terms within a continuum. The data appear to indicate that significant development occurs in the epistemic modal system between the ages of 3 and 4 , and is largely completed by the age of 5 .

\section{Deontic Task}

For the deontic task, two analyses were conducted: one general analysis comparing the two stories where the parents statements coincided and the two where they conflicted, and a second looking at performance on individual stories.

The first analysis considered developmental differences in comprehension of deontic modal expressions comparing the two coincide stories with the two conflict stories. A preliminary analysis showed that the parent who said the statement made no difference in performance, $F(1,51)=0.48$. The mean number of correct predictions after the mothers' statements was 2.93 and after the fathers' statements was 2.83 . Thus, children saw both parents as having similar power. This factor was not considered in further analyses. A 3 (age) $\times 2$ (story type: coincide, conflict) ANOVA revealed main effects of age, $F(2,51)=$ $3.80, p<.03$, and story type, $F(1,51)=20.49, p<.001$. The age $\times$ story type interaction was not significant, $F(1,51)=0.14$. For the main effect of age, the 5 year-olds $(M=6.58)$ performed significantly better than either the 3-year-olds $(M=5.40)$ or 4 -year-olds $(M=5.33)$. The younger two groups didn't differ. Thus, the oldest children were more likely to correctly predict the character's behavior after hearing the deontic expression made by the character's parent.

For the main effect of story type, children performed significantly better on those stories in which what the parents said conflicted $(M=3.30)$ than in which what they said coincided $(M=2.50)$. To consider whether particular stories contributed to this finding, a 3 (age) $\times 4$ (story: "Johnny," "Mary," "Josh," "Sarah") ANOVA was conducted. This analysis revealed the same main effect of age as above, and also a main effect of story, $F(3,153)=21.76, p<.001$. The age $\times$ story interaction was not significant.

For the main effect of story, children performed more poorly on the "Johnny" story than on any other story. The means for the other stories did not differ. More specifically, children had the most difficulty with the coinciding have to $=$ can't pair $(M=0.88)$, and performed equally well on the coinciding $c a n=d o n^{\prime} t$ have to ( $M 1.61)$, conflicting have to versus can $(M=1.61)$, and conflicting can versus have to $(M=1.69)$ pairs.

The significant age effect and the lack of an age $\times$ story interaction implies that children at all ages had particular difficulty with the "Johnny" story. What is interesting about this finding is that the have to $=$ can't pair was the easiest pair on the epistemic task. We considered whether this surprising pattern reflected the fact that subjects were making predictions of behavior on the basis of the character's desire rather than on the parent's statement. If this were true, the following would be the case: (a) on the pair where the character's desire went 
against both parent's statements ("Johnny'), performance would be poorest, yielding a mean close to 0 ; (b) on the pairs where both parents coincide with the child's desire ("Mary"), performance should be best, with a mean close to 2.0; and (c) for those stories in which the parents conflict ("Josh," "Sarah"), performance should be moderately good, with a mean close to 1.0 . However, $t$ tests revealed that the mean for "Johnny" was significantly greater than $0, t(53)$ $=4.44, p<.01$, the mean for "Mary" was significantly less than $2.0, t(53)=$ $3.14, p<.01$, and the means for "Josh" and "Sarah" were significantly greater than 1.0, $t$ 's $(53)=4.71,5.65, p$ 's $<.01$. Thus, it would appear that children were not predicting solely on the basis of the characters' desires. Rather, there would appear to be something particularly compelling about the child wanting to go outside in the "Johnny" story that would cause subjects to predict "went outside." Moreover, the lower performance would have to be a semantic problem rather than a syntactic one because the have to $=$ can't pair was the easiest on the epistemic task.

In sum, whereas age differences did emerge for the deontic task, children performed reasonably well overall. In fact, $35 \%, 27 \%$, and $53 \%$ of the 3-, 4-, and 5-year-olds, respectively, made correct predictions on at least three out of four coincide story trials. The percentages for the two conflict stories were $75 \%$, $80 \%$, and $95 \%$, respectively. The lower percentages for the coincide pairs were almost entirely due to poor performance on the "Johnny" story.

\section{Intertask Analyses}

As a final analysis, we considered whether sentences referring to one of the modal domains (e.g., epistemic) were easier to comprehend than sentences referring to the other modal domain (e.g., deontic). Since sentences were presented singly on the deontic task, the only appropriate comparison would be the singlesentence items of the epistemic task. However, because scores ranged from $0-16$ for the epistemic task and 0-8 for the deontic task, we conducted a 3 (age) $\times 2$ (task: epistemic, deontic) ANOVA on children's scores converted to proportion correct. This analysis revealed significant main effects of age, $F(2,55)=7.61, p$ $<.002$, and task, $F(1,55)=55.12, p<.001$, as well as an age $\times$ task interaction, $F(2,55)=3.42, p<.05$. For the main effect of age, the 5-year-olds were correct on a significantly greater percentage of epistemic single-sentence and deontic trials $(91 \%)$ than either the 4-year-olds $(78 \%)$ or 3 -year-olds $(78 \%)$, who did not differ. However, as can be seen, the younger two groups showed a very high level of success on these single-sentence items. For the main effect of task, children were correct on a significantly greater percentage of epistemic single-sentence items $(95 \%)$ than deontic task trials $(71 \%)$. Analysis of the age $\times$ task interaction showed that whereas the 5-year-olds performed equally well on both tasks, the younger two groups performed significantly better on the epistemic task than deontic task. Also, whereas there were no age differences for the epistemic task single-sentence items, the 5-year-olds performed significantly better than the younger two groups on the deontic task trials. 
It would appear, then, that predicting the location of a penny from a modal expression serving as a guide is an easier task than predicting the behavior of a child story character. Thus, performance on modals is domain-specific, as indicated by the correlation of $r=.10$ between epistemic single-sentence items and deontic task items.

\section{DISCUSSION}

The present study assessed whether preschoolers understand that modal expressions are used both to indicate a speaker's subjective assessment of personal knowledge, and to regulate behavior. We were specifically interested in whether children could use modal expressions to help them guide their search for a hidden object (epistemic modality) and to predict the actions of a story character (deontic modality). Additionally, we considered whether children could make distinctions between degrees of conviction, and understood the effect that negation has on modal expressions. We modified Hirst and Weil's (1982) procedure as a means of demonstrating comprehension of these aspects in preschoolers and considered whether the acquisition pattern for the modal system mirrored that for temporal adverbs and antonyms.

There were two primary findings in the present study. The first is that we revealed substantial knowledge of modal expressions in even our 3- and 4-yearolds. This is particularly true for those trials that presented modal expressions individually. Children's high level of performance on these items demonstrates that, at the very least, they can use surface changes in linguistic form to determine a speaker's subjective assessment of the likelihood of some proposition. Thus, they know that have to and might place one on the belief side of the continuum (favoring location X) and that can't and might not place one on the disbelief side of the continuum (favoring location not- $X$ ). It is clear, then, that even 3-year-olds can combine negation and some modal term to help them guide their search for a hidden object or predict the behavior or a story character.

Development occurred largely in the ability to make fine distinctions between terms that fall on the same side of the continuum; that is, in the ability to make judgments of relative strength. Here, 4- and 5-year-olds performed significantly better than 3-year-olds. Additionally, children found it easier to make a distinction between has to and might as to which indicated more strength in the belief direction, than between can't and might not as to which indicated more strength in the disbelief direction. Thus, it would appear that children first construct a dichotomy between belief and disbelief, and then refine this dichotomy into a continuum with development. A dichotomy would provide the cognitive basis for knowing which terms signify belief in what is stated (have to, might) and which signify disbelief (can't, might not). In order to comprehend the distinction between terms on the same side of the belief-disbelief dichotomy, a continuum needs to be constructed. This acquisition pattern of developing from having partial meaning of modal terms to acquiring the complete modal system does, 
then, seem to mirror that for the antonym and temporal adverb systems (cf. Clark, 1972; Kuczaj, 1975).

This account is supported by the finding for synonymous pairs in which there were no developmental differences for knowing that sentences such as "It has to be under the red cup" refer to the same location as "It can't be under the blue cup. " Technically, comprehension of the symmetrical opposites has to and can't really may only require that a dichotomy or a single opposite relation be constructed. In contrast to these terms, there was developmental change for synonymous sentence pairs such as "It might be under the red cup" and "It might not be under the blue cup." These terms mark a relative degree of conviction. Understanding the notion of relative conviction, however, requires the construction of a continuum. Thus, the findings for the synonymous pairs complement and extend those for the strength contrast pairs.

The second primary finding was that children appeared to have an easier time using modal expressions to help them guide their search (epistemic task) than to help them predict a story character's behavior (deontic task). This would run counter to the claim that, analogous to the history of modal terms in English, children comprehend deontic uses of modal terms before they extend these terms to the epistemic domain (Shepard, 1982). More specifically, it has been claimed that children learn modals first in the deontic domain since the regulation of their behavior is so salient to them. This knowledge is said to be eventually extended by analogy to the epistemic domain. While one could take the position that Shepard's (1982) account is wrong given our data, we argue on the contrary that our findings cannot resolve the issue for several reasons. First, children rarely gave an "I don't know" or irrelevant response when asked to predict the story character's behavior. Instead, they systematically responded with the opposite of what the correct response was, in spite of the character's cited desire. For example, after hearing "You have to stay in" in the "Johnny" story, children who erred typically said "He went outside" when asked to predict. This implies a rebellion strategy rather than a misunderstanding of the modal utterance. In support of this claim, one subject said "He went outside anyway" and another said "He sneaked out." Similarly, for the sentence "You can stand up," many children said "She sat down" even though subjects are told in the story that the character wanted to stand. However, a rebellion strategy largely entails full comprehension of the modal expression; that is, in order to do the opposite of whatever a parent says, one has to know what the parent's statement means. In fact, these findings suggest the prominence of opposites in the modal system for children. Since we cannot tell in the majority of cases whether children were miscomprehending or using a rebellion strategy, we cannot say whether the difference in performance between tasks would be reduced or eliminated under other circumstances. Additional studies that can somehow get around this problem are needed to resolve the issue.

In sum, our use of more familiar modal terms, single-sentence trials, and 
synonymous expressions revealed a high level of comprehension of modal expressions in even our youngest subjects. Consistent with Shatz, Wellman, and Silber's (1983) study of spontaneous productions of mental verbs, our preschoolers evidenced knowledge of how modals are used to indicate a speaker's assessment of what he or she knows. Such facility with modals strongly implies that preschoolers have self-reflective or metacognitive ability not often attributed to them. These findings contradict, then, Pieraut-LeBonniec's (1980) claim that comprehension of possibility and necessity is not apparent prior to age 9 or 10 . Additionally, the continuum paradigm proved to be a highly successful framework for guiding the construction of items and for explaining developmental trends. It appears to have heuristic value for subsequent research on children's comprehension of necessity and possibility.

\section{REFERENCES}

Bymes, J.P., \& Duff, M.A. (1988). Children's comprehension of causal expressions. Child Study Journal, 18, 101-119.

Byrnes, J.P., \& Overton, W.F. (1986). Reasoning about certainty and uncertainty in concrete, causal and propositional contexts. Developmental Psychology, 22, 793-799.

Clark, E.V. (1972). On the child's acquisition of antonyms in two semantic fields. Journal of Verbal Learning and Verbal Behavior, 11, 750-758.

Flavell, J.H. (1985). Cognitive development. Englewood Cliffs, NJ: Prentice-Hall.

Fletcher, P. (1985). A child's learning of English. Oxford: Blackwell.

Gleason, J., Berko, Perlmann, R.Y.. \& Greif, E.B. (1984). What's the magic word: Learning language through politeness routines. Discourse Processes, 7, 493-502.

Grice, H.P. (1975). Logic and conversation. In P. Cole \& J. Morgan (Eds.), Syntax and semantics (Vol. 3, pp. 41-58). New York: Academic.

Hirst, W., \& Weil, J. (1982). Acquisition of epistemic and deontic meanings of modals. Journal of Child Language, 9, 659-666.

Kuczaj, S.A. (1975). On the acquisition of a semantic system. Journal of Verbal Learning and Verbal Behavior, 14, 340-358.

Kuczaj, S.A., \& Maratsos, M.P. (1983). Initial verbs of yes-no questions: A different kind of general grammatical category. Developmental Psychology, 19. 440-444.

Lyons, J. (1977). Semantics (Vol. 2, pp. 787-849). Cambridge: Cambridge University Press.

MacWhinney, B., \& Snow, C. (1985). The child language data exchange system. Journal of Child Language, 12, 271-296.

Pieraut-LeBonniec, G. (1980). The development of modal reasoning: Genesis of necessity and possibility notions. New York: Academic.

Scholnick, E.K., \& Wing, C.S. (1988). Knowing when you don't know: Developmental and situational considerations. Developmental Psychology, 24, 190-196.

Searle, J.R. (1983). Intentionality. Cambridge: Cambridge University Press.

Shatz, M., Wellman, H.M., \& Silber, S. (1983). The acquisition of mental verbs: A systematic investigation of the first reference to mental state. Cognition, 14, 301-321.

Shepard, S.C. (1982). From deontic to epistemic: An analysis of modals in the history of English, creoles, and language acquisition. In A. Ahlquist (Ed.), Papers from the Fifth International Conference on Historical Linguistics (pp. 316-323). Amsterdam: Benjamins.

Wright, G.H. von (1951). An essay in modal logic. Amsterdam: North-Holland. 